\title{
The Automated Breast Volume Scanner (ABVS): initial experiences in lesion detection compared with conventional handheld B-mode ultrasound: a pilot study of 50 cases
}

This article was published in the following Dove Press journal: International Journal of Women's Health 10 October 2011

Number of times this article has been viewed

\author{
Sebastian Wojcinski ${ }^{1}$ \\ Andre Farrokh' \\ Ursula Hille ${ }^{2}$ \\ Jakub Wiskirchen ${ }^{3}$ \\ Samuel Gyapong' \\ Amr A Soliman ${ }^{1,4}$ \\ Friedrich Degenhardt ${ }^{1}$ \\ Peter Hillemanns ${ }^{2}$ \\ 'Department of OB/GYN, Franziskus \\ Hospital, Bielefeld, Germany; ${ }^{2}$ Department \\ of OB/GYN, Hannover Medical School, \\ Hannover, Germany; ${ }^{3}$ Department of \\ Radiology, Franziskus Hospital, Bielefeld, \\ Germany; ${ }^{4}$ Department of OB/GYN, Faculty \\ of Medicine, University of Alexandria, \\ Alexandria, Egypt
}

\section{Video abstract}

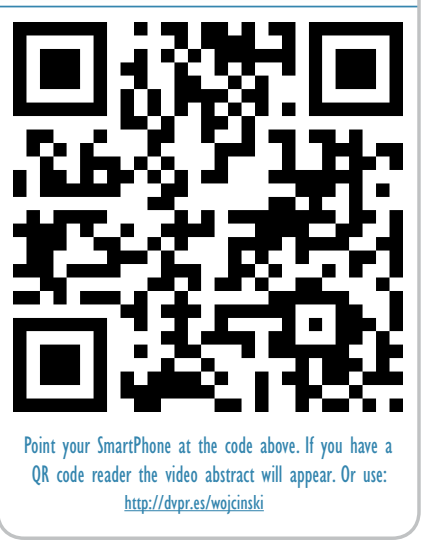

Correspondence: Sebastian Wojcinski Franziskus Hospital, Department of OB/GYN, Kisker Str 27, 336I5 Bielefeld, Germany

Tel +4952I 589 I50 I

$\mathrm{Fax}+49521589 \quad 1504$

Emails@wojcinski.de

\begin{abstract}
The idea of an automated whole breast ultrasound was developed three decades ago. We present our initial experiences with the latest technical advance in this technique, the automated breast volume scanner (ABVS) ACUSON S2000 ${ }^{\mathrm{TM}}$. Volume data sets were collected from 50 patients and a database containing 23 women with no detectable lesions in conventional ultrasound (BI-RADS ${ }^{\circledR}$-US 1), 13 women with clearly benign lesions (BI-RADS ${ }^{\circledR}$-US 2), and 14 women with known breast cancer (BI-RADS ${ }^{\circledR}$-US 5) was created. An independent examiner evaluated the ABVS data on a separate workstation without any prior knowledge of the patients' histories. The diagnostic accuracy for the experimental ABVS was $66.0 \%$ (95\% confidence interval [CI]: 52.9-79.1). The independent examiner detected all breast cancers in the volume data resulting in a calculated sensitivity of $100 \%$ in the described setting ( $95 \%$ CI: $73.2 \%-100 \%$ ). After the ABVS examination, there were a high number of requests for second-look ultrasounds in $47 \%$ (95\% CI: $30.9-63.5)$ of the healthy women (with either a clearly benign lesion or no breast lesions at all in conventional handheld ultrasound). Therefore, the specificity remained at 52.8\% (95\% CI: 35.7-69.2). When comparing the concordance of the ABVS with the gold standard (conventional handheld ultrasound), Cohen's Kappa value as an estimation of the inter-rater reliability was $\kappa=0.37$, indicating fair agreement. In conclusion, the ABVS must still be regarded as an experimental technique for breast ultrasound, which definitely needs to undergo further evaluation studies.
\end{abstract}

Keywords: breast cancer, automated breast ultrasound, automated breast volume scanner, ABVS

\section{Introduction}

Breast ultrasound is a commonly accepted and reliable diagnostic method for women with clinically or radiologically suspicious breast lesions. ${ }^{1}$ Although it is not established as a routine part of screening programs, bilateral whole breast ultrasound has even demonstrated diagnostic advantages in screening asymptomatic women. ${ }^{2-6}$ Breast ultrasound has been performed for more than 50 years. ${ }^{7,8}$ Conventional handheld ultrasound (HHUS) is the gold standard for performing the examination. Obviously, sonography is an examiner-dependent method and the examiner has to be present at the time of image acquisition.

The concept of automated breast ultrasound dates back to the 1970s when the first applicable systems were reported by Maturo et al. ${ }^{9}$ In the current report we present our initial experiences with the latest technical advance in automated breast ultrasound, the Automated Breast Volume Scanner (ACUSON S2000 ${ }^{\mathrm{TM}}$ ABVS; Siemens Medical 
Solutions, Inc, Mountain View, CA). This system can be operated by an assistant medical technician or radiographer. The ABVS acquires a whole series of consecutive B-mode pictures and reconstructs $3 \mathrm{D}$ data sets of the entire breast volume. These data can be sent to a separate workstation to be independently analyzed by a specialist.

We evaluated whether or not breast lesions, previously detected by means of conventional ultrasound, could also be detected and correctly classified by an independent examiner who used only ABVS data. To the best of our knowledge, this is one of the first descriptive studies dealing with the clinical application of the ABVS system.

\section{Materials and methods General design and creation of the patient database}

Our study was conducted at the Breast Cancer Center of Franziskus Hospital in Bielefeld, Germany, between March 2010 and May 2010. The responsible ethical committee did not require additional approval for this noninterventional study design. All examinations were performed using the Siemens ACUSON S2000 ${ }^{\mathrm{TM}}$ ultrasound system (Siemens Medical Solutions, Inc, Mountain View, CA).

The study cohort was recruited from patients who attended the outpatient service of our breast cancer center. The patients were referred to our clinic due to specific diagnostic queries such as palpable breast lesions, breast pain, suspicious mammograms, and intensified screening in highrisk populations. All patients received conventional HHUS as the standard diagnostic method and subsequent examinations whenever necessary. For each patient, the B-mode ultrasound pictures were categorized according to the Breast Imaging Reporting and Data System criteria of the American College of Radiology (ACR BI-RADS ${ }^{\circledR}$-US). ${ }^{10}$

Patients with a final categorization of BI-RADS ${ }^{\circledR}$-US 1, 2 , and 5 in the conventional ultrasound examination were regarded as being suitable for our study. We excluded patients with BI-RADS ${ }^{\circledR}$-US 0, 3 and 4 lesions as the focus of our study was on the bare detection of clearly benign and clearly malignant lesions and not on the evaluation of questionable lesions.

Patients with a history of breast surgery, bra cup sizes greater than D, inflammatory conditions of the breast, and skin disorders were also excluded.

Patients who met the inclusion criteria and who agreed to be enrolled in our study signed an informed consent form and subsequently received the additional ABVS examination.
Thus, we created a database containing 50 patients exhibiting BI-RADS ${ }^{\circledR}$-US 1,2 , and 5 findings in conventional examinations. In our cohort the age ranged from 32 to 72 years (median 52 years). According to the BI-RADS ${ }^{\circledR}$-US categorization, $46 \%(n=23)$ of our cases were assigned as BI-RADS $^{\circledR}$-US 1, 26\% $(n=13)$ had BI-RADS ${ }^{\circledR}$-US 2 lesions, and $28 \%(n=14)$ of the cases had BI-RADS ${ }^{\circledR}$-US 5 lesions. All cases of BI-RADS ${ }^{\circledR}$-US 5 were histologically confirmed after the study setting was completed.

The mean tumor size for the malignant lesions was $23.4 \mathrm{~mm}$ (range 13 to 55 ).

After anonymization, the ABVS database was then available for evaluation by an independent second examiner. We compared the performance of the ABVS (experimental method) to the HHUS (gold standard).

\section{Technical background of the ABVS}

The ACUSON S2000 ${ }^{\mathrm{TM}}$ ABVS is an ultrasound system that automatically surveys and acquires full-field volumes of the breast (Figure 1).

For automated scanning, we used the integrated Siemens 14L5BV linear transducer (14 MHz, $15.4 \mathrm{~cm})$ with 768 piezoelectric elements. These images have a depth of up to $6 \mathrm{~cm}$. In the process of automated scanning, the transducer covers a distance of $16.8 \mathrm{~cm}$, acquiring 318 high-resolution slices for post-processing. Therefore, the maximum volume is $1552.3 \mathrm{~cm}^{3}$ and the slice thickness is about $0.5 \mathrm{~mm}$.

In order to optimize the ABVS results there is a wide range of known imaging modes including tissue harmonic imaging (THI) and Advanced SieClear ${ }^{\mathrm{TM}}$ spatial compounding and Dynamic TCE ${ }^{\mathrm{TM}}$ (tissue contrast enhancement) technology,

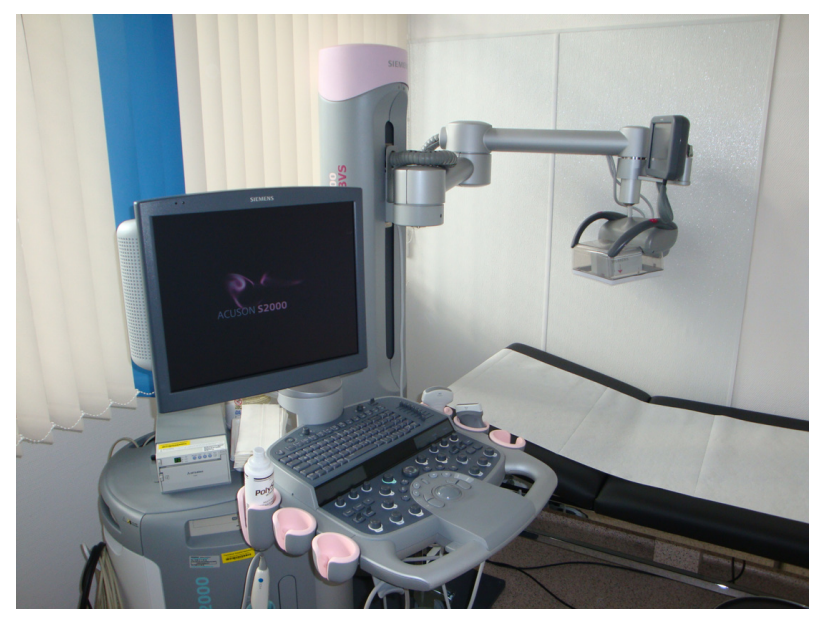

Figure I Installation of the ACUSON S2000 ${ }^{\mathrm{TM}}$ ABVS ultrasound system. On the left-hand side is the ACUSON S2000 ${ }^{\mathrm{TM}}$ machine, on the right-hand side is the I4L5BV volume transducer attached to a mechanical arm. 
as well as new processing algorithms for nipple shadow and reverberation artifacts that are automatically applied when using the ABVS. The Reverberation Removal Algorithm processes the $3 \mathrm{D}$ data and determines whether tissue contact is present and where it is not. The data corresponding to the area with no tissue contact are removed. This is intended to suppress the reverberation artifacts from the noncontact areas. The adaptive Nipple Shadow Reduction Tool analyzes data on a case-by-case basis and is thought to enhance the structures in the retro-areolar area and to improve the visualization of this important region. Finally, a Gain Collection Algorithm analyzes the 3D data and adjusts for the brightness variation artifacts caused by transducer channel-to-channel variations.

After acquisition, the data are automatically sent from the ultrasound system to a breast ultrasound review workstation, which provides comprehensive analysis and manipulation of the 3D data: the workstation presents images through multiplanar reconstruction (MPR, Figure 2). All volume data sets can also be viewed in multiple orientations, including the standard transverse, sagittal, coronal, radial, and anti-radial planes, as well as any other user-defined plane (Figure 3). Secondary images are reconstructed from the acquisition volume in any plane in real-time (Figure 4). A standardized scanning technique, which was also used in our study, has been described elsewhere. ${ }^{11}$

\section{Standard HHUS examination (definition of the gold standard)}

Clinical history taking and clinical examination, and the HHUS, were performed by the author SW, a DEGUM (Deutsche Gesellschaft für Ultraschall in der Medizin, German society for ultrasound in medicine) level II certified senior consultant in gynecology with 7 years' experience in breast ultrasound. ${ }^{12}$ This examiner also knew the results of

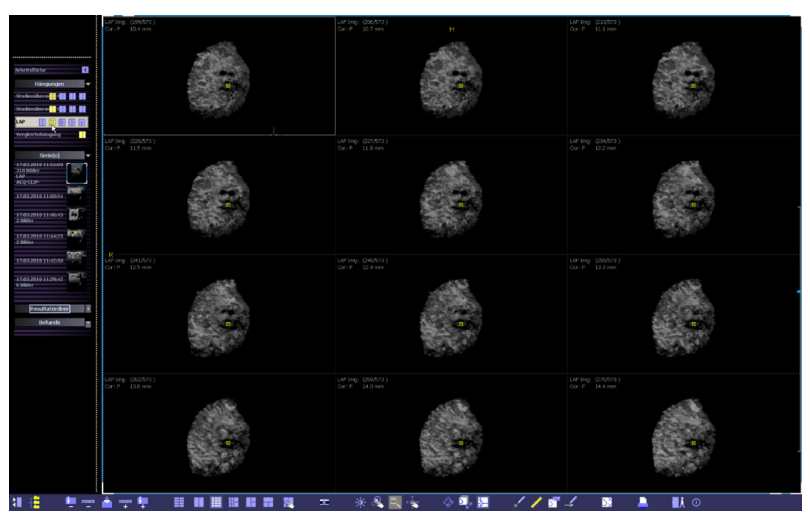

Figure 2 Multi-planar reconstruction of the volume data displayed on the automated breast volume scanner workplace.

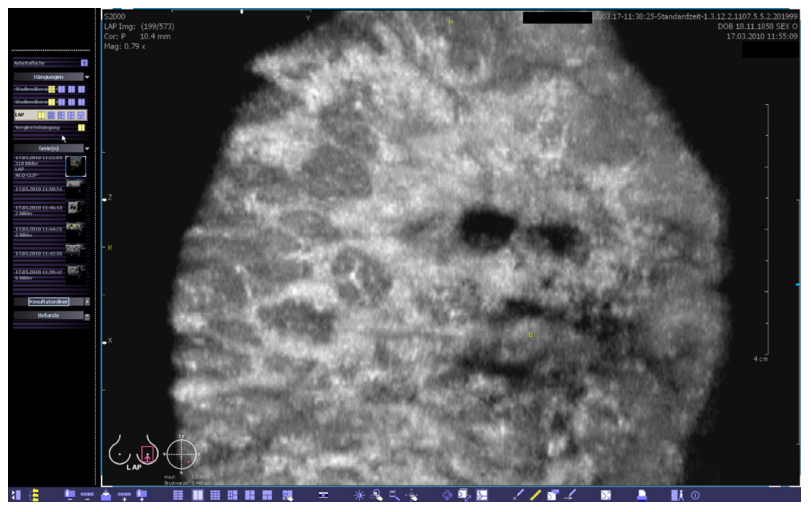

Figure 3 Coronal view of the volume data at a depth of $10.4 \mathrm{~mm}$ from the skin line. The yellow spot indicates the position of the nipple.

the other imaging modalities when available (mammography, magnetic resonance imaging [MRI]).

For HHUS we used the integrated Siemens 18L6 HD linear transducer $(5.5-18 \mathrm{MHz}, 5.6 \mathrm{~cm})$. The patient was in the supine position with the ipsilateral hand raised above the head. All examinations were performed with the ultrasound probe oriented perpendicular to the chest wall. During each examination, all necessary B-mode pictures were first obtained according to the diagnostic standards. All images were digitally recorded. The examiner categorized the breast lesions detected according to the ACR BI-RADS ${ }^{\circledR}$-US classification system. These results were defined as the gold standard (reference standard) for comparison with the experimental method. All standard examinations were completed before the patients were enrolled in the study. Eligible patients were subsequently examined using the ABVS.

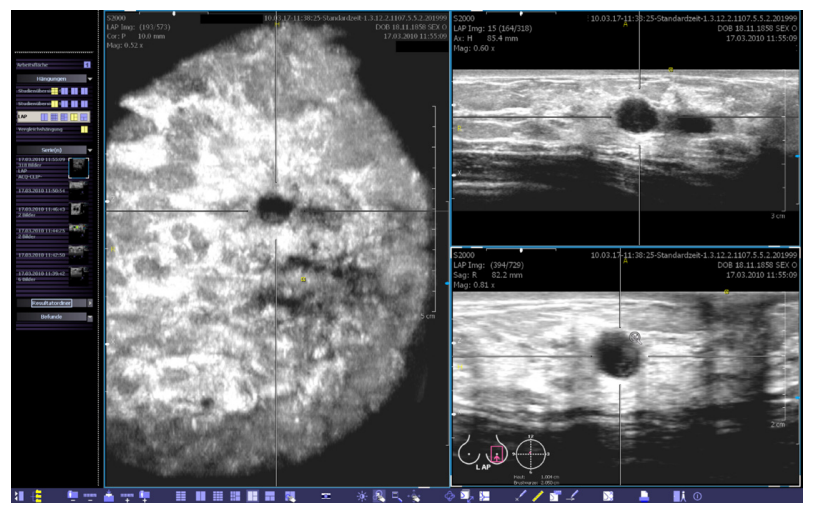

Figure 4 This view provides the coronal (left), transverse (upper right) and sagittal (lower right) planes. The body marker indicates that this volume was acquired at the apex of the left breast. The yellow spot marks the position of the nipple. A point of interest can be chosen and marked by two orthogonal lines. Then, the corresponding cross-sections are calculated in real-time and shown in the other planes. The images can be optimized by adjusting the magnification, brightness, and contrast. 


\section{Acquisition of the ABVS data}

The experimental ABVS examination was also performed by the author, SW. For the ABVS examination, the patient was placed in the same position as for the HHUS. A special ultrasound lotion that provides optimal imaging results in combination with the ABVS was applied to the skin instead of the usual ultrasound gel (Polysonic Ultrasound Lotion, Parker Laboratories, Inc, Fairfield, NJ).

According to other studies, we used the bra cup size to describe the size of the breast. ${ }^{13}$ Bra cup size is determined by measuring the horizontal chest circumference in the inframammary fold, adding 5 inches $(12.7 \mathrm{~cm})$ and subtracting the horizontal chest circumference at the fullest part of the breasts. A difference of $1 \mathrm{inch}(2.54 \mathrm{~cm})$ is equivalent to an A cup, 2 inches a B cup, and so on. ${ }^{14}$ We did not perform this measurement in the current study, but instead asked the patients for their bra cup size.

Depending on the bra cup size, the examiner chose the number of scans to be taken from each side. Usually, breasts with A-cup and B-cup sizes can be fully displayed by performing two volume scans (medial and lateral, Figure 5). In breasts with C-cup or D-cup sizes it is often necessary to choose additional views (usually a separate view of the apex and the axillary process of the breast, Figure 5).

As standard, there is a replaceable membrane fixed to the transducer to assure sufficient contact with the skin of the entire area. In order to acquire the ultrasound image volume, the 14L5BV transducer was positioned on the breast with slight pressure. The mechanical arm that is part of the scanner column was locked in the chosen position. A preset scanning program was chosen according to the bra cup size of the breast. In addition, all previously known ultrasound features were available in order to optimize picture quality. The automated scan took between 55 and 65 seconds. There was no need for breath holding during this time. After a particular scan was completed, the coronal plane of the volume acquired was displayed at the height of skin level. This enabled the examiner to set a fixed marker on the nipple, allowing orientation in the

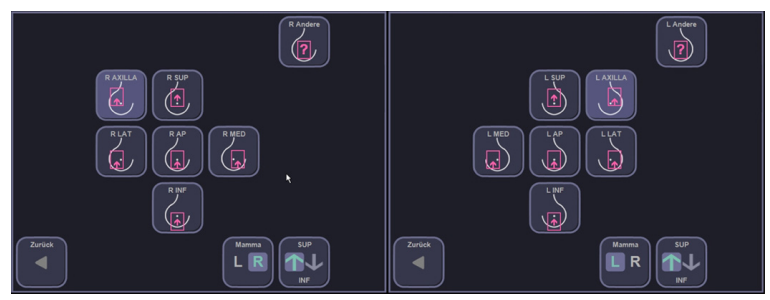

Figure 5 Predefined positions of the scanner, which are used to cover the entire volume of an individual breast. subsequent analysis. Finally, the entire set of volume scans was sent to the workstation.

\section{Independent interpretation of the experimental ABVS data (by examiner 2)}

The independent interpretation of the ABVS data sets was performed by the co-author AF, a DEGUM level I certified senior resident in gynecology with 4 years' experience in breast ultrasound.

The second examiner exclusively analyzed the 3D data sets without prior knowledge of the patients' histories, clinical findings, or results of the other imaging modalities. The second examiner had no information about the proportion of BI-RADS ${ }^{\circledR}$-US 1,2 , and 5 cases in the database, but he did know that BI-RADS ${ }^{\circledR}$-US 3 and 4 cases had been excluded.

This examiner was able to use a variety of tools for image manipulation, including the standard views (axial, sagittal, coronal, radial, anti-radial), user-defined views, rotation around $\mathrm{x}, \mathrm{y}, \mathrm{z}$ axes, free rotation around any point of interest, a magnifier and interactive zoom, marking and annotation of areas of interest, and the generation of snapshots.

The second examiner used the following standard procedure to analyze the ABVS data sets: first, the whole volume was analyzed in the coronal plane moving slowly from the skin to the chest wall. Suspicious lesions were marked with the system's default tool. In the next step, all lesions were evaluated by generally re-examining them in the sagittal and axial planes (and optionally in any other plane), using adequate magnification, brightness, and contrast. Finally, the examiner moved through the whole volume in the sagittal and axial planes in order to potentially detect additional lesions that were not seen in the coronal plane. Lastly, the examiner assigned the lesions a category according to the ACR BI-RADS ${ }^{\circledR}$-US system.

Despite the fact that the second examiner knew that there were no BI-RADS ${ }^{\circledR}$-US 0,3 , or 4 cases in the database, he was allowed to categorize lesions as BI-RADS ${ }^{\circledR}$-US 0,3 , or 4 whenever he requested a second-look ultrasound in order to scrutinize suspicious lesions.

When a second-look ultrasound was requested for a lesion that eventually turned out to be benign, the result of the AVBS examination was defined as "nonconcordant" and "falsepositive". On the other hand, when a second-look ultrasound was requested for a lesion that turned out to be malignant, the result was classified as "true-positive" because the cancer could then be correctly detected in the subsequent conventional ultrasound. 


\section{Ultrasound quality assurance}

Due to the national regulating authority statutes, all breast ultrasound systems have to fulfill basic technical requirements and undergo regular quality control measures. ${ }^{15}$ The technical requirements include a linear transducer covering a least $38 \mathrm{~mm}$, an ultrasound frequency $\geq 7 \mathrm{MHz}$, an adjustable digital focus, a time gain control signal generator, a frame rate $\geq 15 \mathrm{fps}$, an image depth $\geq 6 \mathrm{~cm}$, the detection of a returning echo $\geq 60 \mathrm{~dB}$ below the transmitted signal, and at least 8-bit processing of the images.

In order to guarantee long-lasting performance of the transducer and conformance of the whole device with the standards, a compliance test is mandatory when setting up a new ultrasound system and a consistency inspection is needed every 4 years. All of the abovementioned standards applied to the equipment used in our study.

\section{Statistical analysis}

The software package SPSS Statistics (v 17.0; SPSS, Inc, Chicago, IL) was used for the statistical analysis. The results obtained from the experimental method (ABVS) were compared with the results from the gold standard (HHUS). The data were analyzed using descriptive statistics. The diagnostic sensitivity and specificity, as well as the accuracy of the ABVS, were calculated based on the Bayesian theorem using Fisher's exact test.

The Chi-square test was used to assess the correlation between the expected and the observed rate of second-look ultrasounds.

Calculation of the concordance with the BI-RADS ${ }^{\circledR}$ classification was based on Cohen's Kappa test. ${ }^{16}$ Although both examiners (for both HHUS and ABVS) had different degrees of information about the patients, this test can provide a satisfactory estimation of the true inter-rater reliability. We used the magnitude guidelines published by Landis and Koch, who characterized the values of $\kappa<0$ as indicating no agreement and $\kappa 0-0.20$ slight, $\kappa$ 0.21-0.40 fair, $\kappa$ 0.41-0.60 moderate, $\kappa$ 0.61-0.80 substantial, and $\kappa$ $0.81-1$ as almost perfect agreement. ${ }^{17}$

Statistical significance was assumed at $P<0.05$ for all tests.

\section{Results \\ Accuracy, sensitivity, and specificity of the ABVS}

The sensitivity of the ABVS in detecting malignant lesions ranged from 73.2 to $100 \%(95 \% \mathrm{CI})$, and all of the 14 cancer patients were detected by the second examiner. The diagnostic accuracy of the experimental ABVS was $66.0 \%$ (95\% CI: 52.9-79.1). The specificity seemed to be quite low at $52.8 \%$ (95\% CI: $35.7-69.2)$ as there was a high number of requests for second-look ultrasounds after the ABVS and these cases are, as a matter of fact, false-positive if there is no cancer. These results must be carefully interpreted, bearing in mind that the experimental ABVS was performed on a subset of patients with results defined by the HHUS.

\section{Rate of second-look ultrasounds}

We expected 14 (28\%) requests for second-look ultrasound after the ABVS examination as there were 14 cases of BI-RADS $^{\circledR}$-US 5 lesions. We did not expect requests for the other 36 cases (BI-RADS ${ }^{\circledR}$-US 1 or 2 lesions). Nevertheless, the observed rate of second-look ultrasounds was significantly high, totaling $62 \%$ (95\% CI: 48.6-75.5, $P<10^{-8}$ ).

Regarding the subgroups, there was a request for a second-look ultrasound in 61.5\% (95\% CI: 35.1-88.0) of the women with benign lesions and even in $39.1 \%$ (95\% CI: 19.2-59.1) of the women with no breast lesions at all. Overall, examiner 2 requested second-look ultrasounds for all 14 cases of breast cancer but also in 17 of the 36 controls (47\% [95\% CI: 30.9-63.5]). These data are shown in Table 1.

Table I Agreement between examiner I (gold standard) and examiner 2 (experimental ABVS data interpretation) focusing on the correct clinical decision of whether the patient should undergo a control ultrasound due to a suspicious finding or whether the patient should be defined as healthy as there is no suspicious lesion

\begin{tabular}{lll}
\hline & $\begin{array}{l}\text { Classification by examiner lusing all information and HHUS } \\
\text { (gold standard) }\end{array}$ & Total \\
\cline { 2 - 3 } & Disease - (BI-RADS I, 2) & \\
\hline Classification by examiner 2 analyzing the & ABVS data (experimental method) & \\
ABVS $-($ BI-RADS I, 2) & 19 & 0 \\
ABVS + (BI-RADS 0, 3, 4, 5) & 17 & 14 \\
Total & 36 & 14 \\
\hline
\end{tabular}

Abbreviations: ABVS, automated breast volume scanner; HHUS, handheld ultrasound. 


\section{Estimation of the inter-rater reliability}

We compared the results of the BI-RADS ${ }^{\circledR}$-US classification by the second examiner, who only had access to the ABVS data but not to any other information, with the results of the first examiner (HHUS, which can be seen as the gold standard). Cohen's Kappa value as an estimation of the inter-rater reliability regarding this comparison was $\kappa=0.37$ (95\% CI: 0.19-0.55), reaching an agreement that can be described as fair between both modalities. The detailed results are shown in Table 2.

\section{Diagnostic performance in the subgroup of BI-RADS ${ }^{\circledR}$-US 5 lesions}

Focusing on the 14 lesions that were classified as BI-RADS ${ }^{\circledR}$-US 5 in conventional ultrasound, we obtained the following results: eight out of 14 lesions (57\%) were correctly classified as BI-RADS ${ }^{\circledR}$-US 5 , and six out of 14 lesions were classified as BI-RADS ${ }^{\circledR}$-US 0,3 or 4, resulting in a second-look ultrasound being requested by examiner 2 . Therefore, none of the carcinomas would have been missed by the ABVS, as all patients were scheduled for further examinations and none of the 14 patients was classified as BI-RADS ${ }^{\circledR}$-US 1 or 2 , which would have resulted in a delayed diagnosis.

\section{Diagnostic performance in the subgroup of ${\mathrm{BI}-R A D S^{\circledR}{ }_{-} \cup S} 2$ lesions}

With respect to the BI-RADS ${ }^{\circledR}$-US 2 lesions $(n=13)$, we performed a test identical to the BI-RADS ${ }^{\circledR}$-US 5 lesion test. Only four (30.8\%) of these lesions were correctly classified as BI-RADS $^{\circledR}$-US 2. One lesion was missed and the breast was therefore classified BI-RADS ${ }^{\circledR}$-US 1.A second-look ultrasound was requested in eight cases (61.5\% [95\% CI: 35.1-88.0]) as examiner 2 either diagnosed a questionable lesion (six cases) or was suspicious of a malignant lesion ( 2 cases).

\section{Diagnostic performance in the subgroup

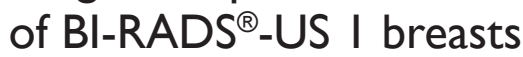

Furthermore, we analyzed the dataset of BI-RADS ${ }^{\circledR}$-US 1 breasts, which exhibited no verifiable lesions $(n=23) ; 13$
(56.5\%) were correctly described as BI-RADS ${ }^{\circledR}$-US 1 . In one case, examiner 2 suspected a benign lesion (BI-RADS ${ }^{\circledR}$-US 2, Figure 6). There were no category BI-RADS ${ }^{\circledR}$-US 5 diagnoses, but nine (39.1\% [95\% CI: 19.2-59.1]) secondlook ultrasounds were requested due to questionable lesions categorized as BI-RADS ${ }^{\circledR}$-US 0,3 or 4.

\section{Experiences in clinical applicability}

The standard examinations, which included history taking, clinical examination, and HHUS, took about 20-30 minutes on average per patient. An additional 8-10 minutes was needed to acquire the ABVS data sets. This extra time period included informing the patient about the procedure, applying the ultrasound lotion, and acquiring two to four image sets per breast. Regarding the size of the breast, there was no difficulty in acquiring the whole breast volume in women with bra cup sizes A to D, but there were noticeable deficits in women with larger breasts. Interpretation times were about 6-10 minutes per patient. However, excessive time had to be spent on the second-look HHUS, as mentioned above.

\section{Clinical examples}

Figure 7 shows a case from the database that was correctly classified as BI-RADS ${ }^{\circledR}$-US 5 (Figure 7). The 53-year-old patient was asymptomatic and had had a screening mammogram 6 months earlier with no evidence of a malignant breast lesion. The breast density had been categorized as ACR III. The patient reported menopause one year earlier. She had given birth to three children (first child at the age of 20 years), but there was no history of breast feeding. Her body mass index was 21.4 at the time of presentation and her bra cup size was B. The patient reported no hormone replacement therapy and no other relevant intake of medications. There was no family history of cancer. The patient was sent to our breast cancer center because of a suspicious finding in a conventional breast ultrasound that had been performed for screening purposes. The lesion remained occult in the directly repeated mammogram. The occult lesion could also be easily detected by the ABVS system.

Table 2 Agreement between examiner I (gold standard) and examiner 2 (experimental ABVS data interpretation) focusing on the correct classification according to the ACR BI-RADS ${ }^{\circledR}$-US system

\begin{tabular}{|c|c|c|c|c|c|}
\hline & \multicolumn{4}{|c|}{ Classification by examiner I using all information and HHUS (reference standard) } & \multirow[t]{2}{*}{ Total } \\
\hline & BI-RADS I & BI-RADS 2 & BI-RADS 0, 3, 4 (excluded) & BI-RADS 5 & \\
\hline \multicolumn{6}{|c|}{ Classification by examiner 2 analyzing the ABVS data (experimental method) } \\
\hline BI-RADS I & 13 & 1 & 0 & 0 & 14 \\
\hline BI-RADS 2 & I & 4 & 0 & 0 & 5 \\
\hline BI-RADS $0,3,4$ & 9 & 6 & 0 & 6 & 21 \\
\hline BI-RADS 5 & 0 & 2 & 0 & 8 & 10 \\
\hline Total & 23 & 13 & 0 & 14 & 50 \\
\hline
\end{tabular}

Abbreviations: ABVS, automated breast volume scanner; HHUS, handheld ultrasound. 


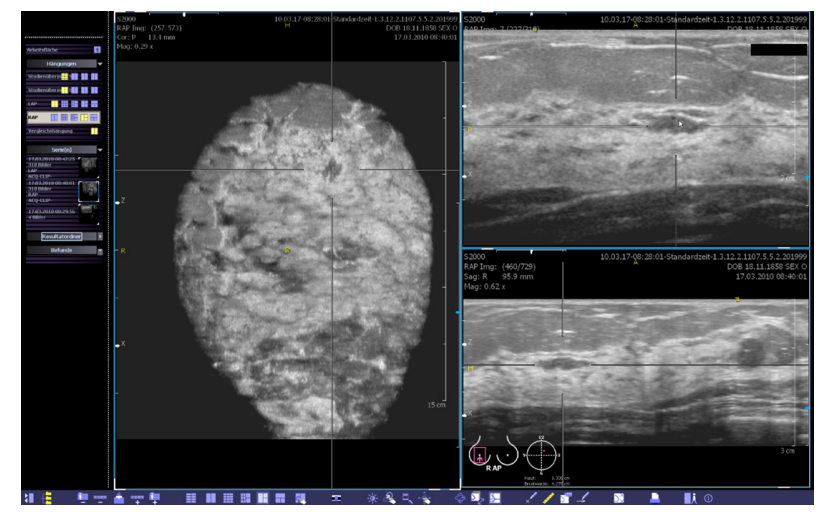

Figure 6 Case from the database demonstrating a BI-RADS ${ }^{\circledR}-$ US I breast that was interpreted as a BI-RADS ${ }^{\circledR}$-US 2 lesion, mainly visible in the coronal plane by examiner 2 .

We would like to present three further clinical examples that were not included in our database but which illustrate the diagnostic imaging capabilities of the automated ultrasound system. Although there is no superiority of the ABVS over HHUS in these cases, there might be hints for the future implementations of the ABVS as a useful method in breast diagnostics.

Figure 8 shows a 50 -year-old patient with known multifocal breast cancer. We used the ABVS system to exactly locate the
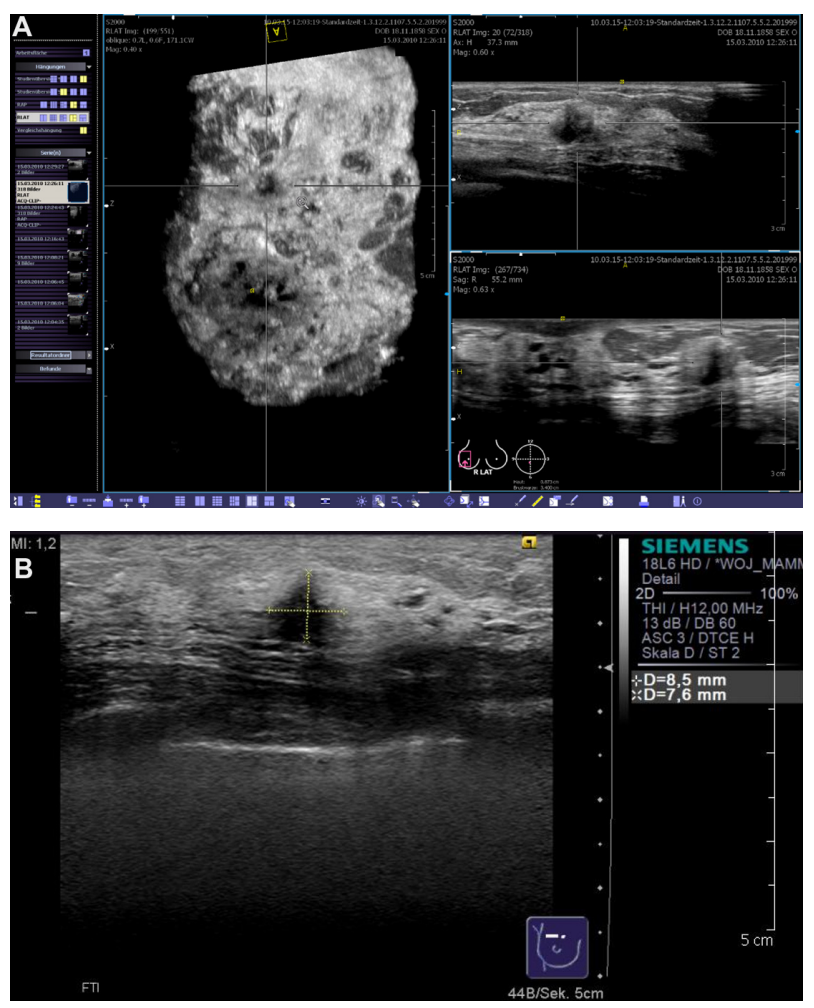

Figure 7 Case from the database demonstrating a BI-RADS ${ }^{\circledR}$-US 5 lesion that remained occult in the mammogram. The lesion was easily detected in the volume data set by examiner 2 (A) and a second-look ultrasound (B) with histological confirmation was correctly requested.
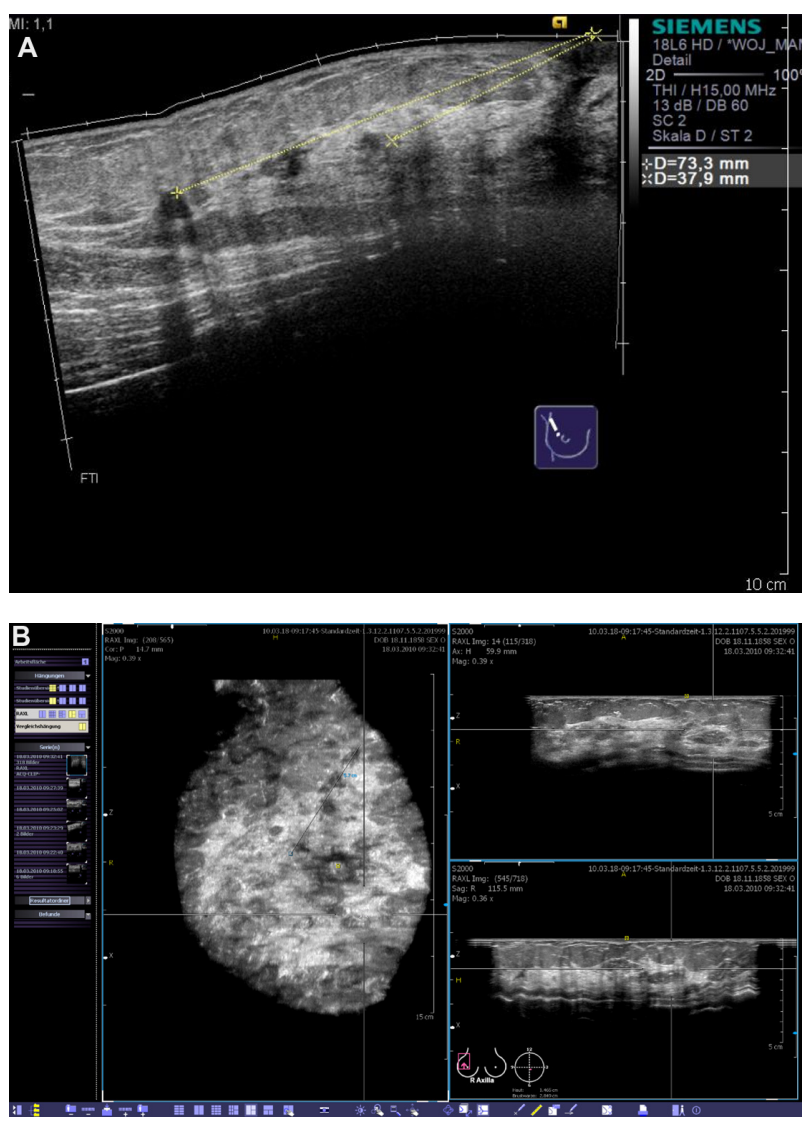

Figure $\mathbf{8}$ Clinical example of multifocal breast cancer. With conventional ultrasound, even when using techniques such as SieScape ${ }^{\circledR}$ panoramic imaging, it is difficult to display more than three lesions at the same time (A). In the coronal plane, derived from the volume data sets, all foci can be viewed at the same time (B). While remaining aware of the extension of tissue changes, we planned a quadrantectomy after wire localization.

four invasive lesions in order to get an idea of the required resection volume. Finally, we performed a quadrantectomy after wire localization of the superior and inferior limits of the area. The histological results revealed clear resection margins for all four lesions. Due to the 3-dimensional planning of the surgical procedure, an acceptable cosmetic result was achieved.

Figure 9 shows the case of a 47 -year-old patient with a locally advanced invasive lobular carcinoma of the right breast. The dimension of the lesion was poorly seen in the mammogram and the lesion was too extensive for distinct measurement or localization in the conventional ultrasound. Therefore, we ordered an MRI as well as an ABVS examination. In the coronal plane derived from the ABVS 3D data set, we were able to detect the lesion easily, with its associated tissue distortion and spiculations, enabling us precisely to perform a tumor-adapted reduction mammaplasty. An excellent correlation was found between the suspected dimensions of the lesion in the MRI and the ABVS compared with the histological results. 


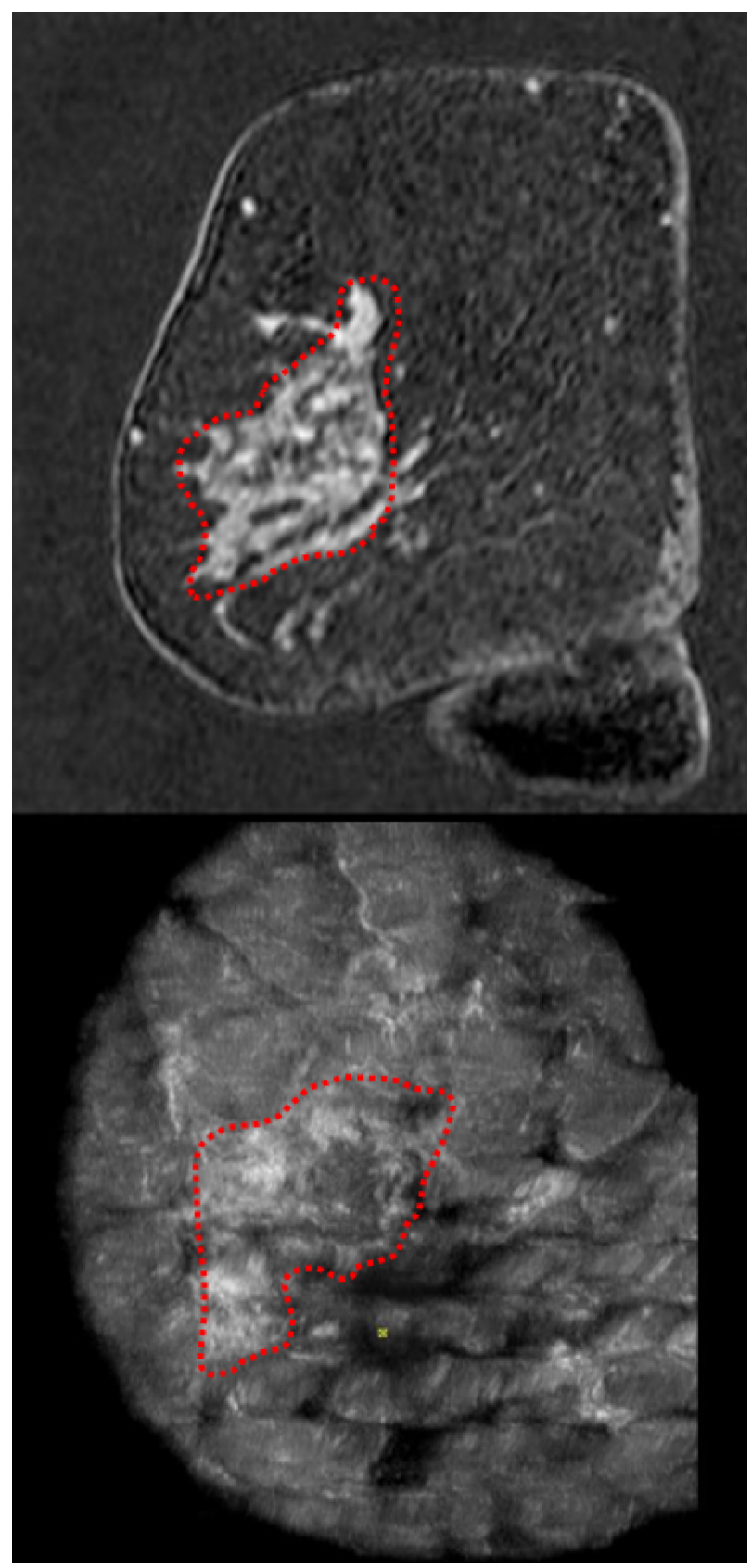

Figure 9 Clinical example of an invasive lobular breast cancer. In order to estimate the extension of the lesion, we performed both MRI and the automated breast volume scanner. The coronal planes of both imaging modalities demonstrated a correlation in the measurements of the lesion.

Finally, we present the case of a 53-year-old patient who presented to us with bloody nipple discharge (Figure 10). Before any manipulation of the breast by a clinical examination, galactography, or conventional ultrasound, we performed an ABVS examination. A pathological duct ectasia at the 9 o'clock position was clearly displayed in the coronal plane. Upon reconstructing the other planes we suspected an intraductal proliferation. Galactography was performed that confirmed the diagnosis of intraductal proliferation. Cytological
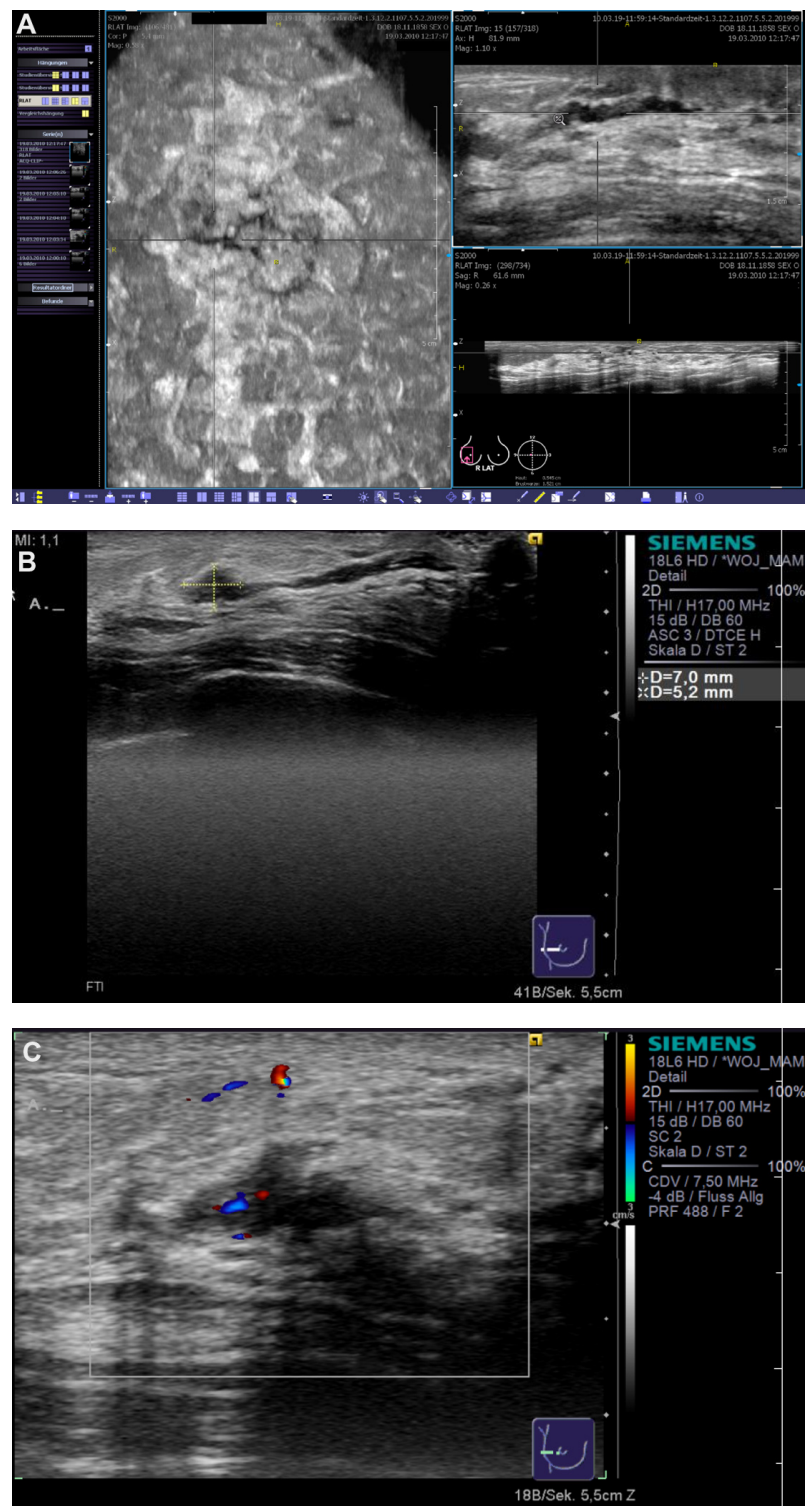

Figure 10 Clinical example of a patient with nipple discharge. The duct ectasia can be seen in the reconstructed coronal plane (A, left), and an intraductal lesion was suspected in the transverse (A, upper right) and sagittal planes (A, lower right). Conventional ultrasound (B) confirmed the diagnostic findings of a vasculated (C) papillary lesion.

examination of the discharge yielded no additional information. Therefore, a ductectomy was performed and the final histological diagnosis was a benign intraductal papilloma.

\section{Discussion}

Breast ultrasound, as currently considered among breast imaging modalities, has an essential and specific role as a complementary method to mammography, by adding to the diagnostic accuracy. Breast ultrasound is an accepted and well-established diagnostic tool for women with clinically or radiologically detected suspicious breast lesions. ${ }^{1}$ Handheld ultrasound represents the gold standard for this examination. 
The concept of breast cancer diagnosis using an automated ultrasound system must be regarded as an experimental approach. Nevertheless, the general idea is not new and the technical equipment has evolved over the last three decades. ${ }^{18-20}$ However, automated whole breast scanning is far from representing an accepted medical practice and its application still lacks solid data from prospective studies.

We present our initial experiences with the latest generation of an automated breast ultrasound system, the Automated Breast Volume Scanner ACUSON S2000 ${ }^{\mathrm{TM}}$ ABVS (Siemens Medical Solutions, Inc, Mountain View, CA).

In a clinical setting, use of the ABVS is a feasible method that can be easily integrated into the workflow of a breast cancer center. As the probe is equipped with 768 piezoelectric elements and reaches $14 \mathrm{MHz}$, we found sufficient image quality and resolution. The ABVS guarantees high patient safety as there is no exposure to ionizing radiation and no injection of contrast medium.

The ABVS image data provide the following additional information to the examiner: the coronal view of the entire volume offers an easily understandable representation of the breast's global anatomy and architecture. In particular, the segmental organization of the ductal system and surrounding tissue is clearly visualized. This view provides physicians and, especially, surgeons a comprehensive sight of the breast from the skin line to the chest wall. In our experience this information helps in better defining the margins for resection in breast cancer surgery.

The HHUS is known to be a time-consuming, examinerdependent, and therefore expensive procedure. At first glance, the ABVS seems to overcome these problems as it can be performed by a medical assistant, it allows a delayed interpretation of the images by an independent examiner at any time, and it provides reproducible, standardized views of the entire breast volume. Indeed, the examination time can initially be reduced from approximately $20-30$ minutes for a full HHUS to $8-10$ minutes with the ABVS. On the other hand, the large number of time-consuming second-look ultrasounds has to be taken into account. In fact, these examinations are performed by conventional HHUS and therefore dissolve any previously assumed advantages regarding time. However, our study was not designed to fully answer the question of examination time.

The reason for the high rate of second-look ultrasounds can be explained by the fact that when the ABVS system is used, we lose the ability to immediately explore further a questionable lesion by modifying factors such as compression, the orientation of the probe, and the machine's setting while acquiring the image in real-time. Ultrasound techniques such as Doppler imaging or sonoelastography cannot be used either. Furthermore, we lack standardized interpretation criteria and there are still technical artifacts in the volume data set. These problems are reflected in the low specificity of 52.8\%. Nevertheless, with accumulating data, growing experience, standardized diagnostic criteria, and optimized techniques, this specificity might be improved.

In our preselected database we apparently showed a high sensitivity to the method (nominally $100 \%$ [95\% CI: $73.2-$ 100]), as no case of breast cancer was missed or misinterpreted as a benign lesion. These results must definitely undergo further confirmation as there were several limitations to our study.

The main limitation to our study was the design with a limited sample size of only 50 preselected patients. The proportion of cases to controls was not representative of the whole population. Therefore, the results concerning sensitivity, specificity, and the rate of second-look ultrasounds cannot be applied to the general population and hence must be carefully interpreted. We present our work only as a pilot study with a completely new ultrasound system.

With a more representative cohort and with even more experience in interpreting the volume data sets, we would expect to find a lower number of requests for second-look ultrasounds and improved specificity, but also a lower sensitivity. Other limitations included the small sample size, resulting in vast confidence intervals, and the unicentric nature of the study. As previously mentioned, the technique itself is limited to women with breast sizes up to a bra cupsize $\mathrm{D}$. In addition, there is no experience in examining the axillary region with the ABVS, although this region is of special importance in breast cancer diagnostics.

In order to move this technique forward, there is a definite need for further research. In particular, we require prospective studies recruiting larger patient cohorts. This would involve a multi-center design and could also include a multi-observer analysis. We propose two general designs for subsequent high-level studies:

1. Patients could be recruited based on the initial mammography or ultrasound BI-RADS ${ }^{\circledR}$ categorization. This setting would yield information about the ABVS as an adjunct to the conventional technique for these special groups of patients (eg, detecting previously occult cancers in women with a primary categorization of BI-RADS ${ }^{\circledR} 1$ or 2 ; further describing a lesion in women with BI-RADS ${ }^{\circledR} 3$ or 4 ; finding additional lesions in women with BI-RADS ${ }^{\circledR}$ 5).

2. The ABVS could also be used as the sole examination technique on a randomly assigned cohort of asymptomatic 
women. The cohort could then be compared with agematched controls who are examined by the conventional techniques. This setting would yield data about the performance of the ABVS as an ultrasound-based screening tool (eg, in the general population, in young women with dense breasts, in high-risk populations, and for detecting small lesions, for example).

\section{Summary}

As there is a lack of definite data, the ABVS should be used only within approved study protocols. So far, we do not recommend integrating the ABVS into routine diagnostic procedures, neither as an adjunct nor as an alternative for HHUS.

In the future, the ABVS might demonstrate greater usefulness with further improvements in technology and when the distinct diagnostic criteria have become standardized. Currently, whether or not there will be an improvement in cancer detection or a reduction in unnecessary biopsies when using the ABVS as a diagnostic modality, remains unclear.

Appropriate clinical indications for this examination need to be developed and this method must undergo standardized imaging methodology evaluation and validation studies. Therefore, currently, the ABVS should remain a topic of future discussion and research within the proposed study designs.

\section{Authors' contributions}

SW and AF contributed to the conception and design of the study and performed the ultrasound examinations, data collection, and analysis. SG, UH, and PH contributed to the statistical analysis, a more advanced interpretation of the data and the writing and reviewing of the manuscript. JW provided the radiological material and reviewed the radiological report data. FD and AS conducted final reviews of the database and the manuscript and FD provided methodological advice.

\section{Disclosure}

The authors report no conflicts of interest in this work.

\section{References}

1. Zonderland HM, Coerkamp EG, Hermans J, van de Vijver MJ, van Voorthuisen AE, et al. Diagnosis of breast cancer: contribution of US as an adjunct to mammography. Radiology. 1999;213:413-422.
2. Buchberger W, Niehoff A, Obrist P, et al. Clinically and mammographically occult breast lesions: detection and classification with high resolution sonography. Semin Ultrasound CT MR. 2000;21:325-336.

3. Corsetti V, Ferrari A, Ghirardi M, et al. Role of ultrasonography in detecting mammographically occult breast carcinoma in women with dense breasts. Radiol Med. 2006;111:440-448.

4. Gordon PB, Goldenberg SL. Malignant breast masses detected only by ultrasound. A retrospective review. Cancer. 1995;76:626-630.

5. Kolb TM, Lichy J, Newhouse JH. Occult cancer in women with dense breasts: detection with screening US - diagnostic yield and tumor characteristics. Radiology. 1998;207:191-199.

6. Nothacker M, Duda V, Hahn M, et al. Early detection of breast cancer: benefits and risks of supplemental breast ultrasound in asymptomatic women with mammographically dense breast tissue. A systematic review. BMC Cancer. 2009;9:335.

7. Wild JJ, Neal D. Use of high-frequency ultrasonic waves for detecting changes of texture in living tissue. Lancet. 1951;1:655-657.

8. Wild JJ, Reid JH. Echographic visualization of the living intact human breast. Cancer Res. 1954;14:227-282.

9. Maturo VG, Zusmer NR, Gilson AJ, et al. Ultrasound of the whole breast utilizing a dedicated automated breast scanner. Radiology. 1980;137:457-463.

10. Mendelson EB, Baum JK, Berg WA, et al. BI-RADS: Ultrasound. In: D'Orsi CJ, Mendelson EB, Ikeda DM, et al, editors. Breast Imaging Reporting and Data System: ACR BI-RADS - Breast Imaging Atlas. Reston, VA, American College of Radiology; 2003.

11. Tozaki $M$, Isobe $S$, Yamaguchi $M$, et al. Optimal scanning technique to cover the whole breast using an automated breast volume scanner. Jpn J Radiol. 2010;28:325-328.

12. Deutsche Gesellschaft für Ultraschall in der Medizin (DEGUM). Mehrstufenkonzept Mammasonographie. Available from: http://www. degum.de/Mehrstufenkonzept_Mammasonogra.634.0.html. Accessed July 3, 2011.

13. Kusano AS, Trichopoulos D, Terry KL, Chen WY, Willet WC, Michels KB. A prospective study of breast size and premenopausal breast cancer incidence. Int J Cancer. 2006;118:2031-2034.

14. Wright MC. Graphical analysis of bra size calculation procedures. Int J Cloth Sci Technol. 2002;14:41-45.

15. [German Federal Association of Fund Doctors. Ultrasound regulations]. Available from: http://www.kbv.de/rechtsquellen/2488.html. Accessed July 3, 2011. German.

16. Cohen J. A coefficient of agreement for nominal scales. Educ Psych Meas. 1960;20:37-46.

17. Landis JR, Koch GG. The measurement of observer agreement for categorical data. Biometrics. 1977;33:159-174.

18. Egan RL, Egan KL. Automated water-path full-breast sonography: correlation with histology of 176 solid lesions. AJR Am J Roentgenol. 1984;143:499-507.

19. Kelly KM, Dean J, Lee SJ, Comulada WS. Breast cancer detection: radiologists' performance using mammography with and without automated whole-breast ultrasound. Eur Radiol. 2010;20:2557-2564.

20. Wenkel E, Heckmann M, Heinrich M, et al. Automated breast ultrasound: lesion detection and BI-RADS classification - a pilot study. Rofo. 2008;180:804-808.
International Journal of Women's Health

\section{Publish your work in this journal}

The International Journal of Women's Health is an international, peerreviewed open-access journal publishing original research, reports, reviews and commentaries on all aspects of women's healthcare including gynecology, obstetrics, and breast cancer. Subject areas include: Chronic conditions (migraine headaches, arthritis, osteoporosis);

\section{Dovepress}

Endocrine and autoimmune syndromes; Sexual and reproductive health; Psychological and psychosocial conditions. The manuscript management system is completely online and includes a very quick and fair peer-review system. Visit http://www.dovepress.com/ testimonials.php to read real quotes from published authors. 